\title{
Simultaneous Onset of Ulcerative Colitis and Disseminated Pyoderma Gangrenosum
}

\author{
Albrecht Neesse Patrick Michl Steffen Kunsch \\ Volker Ellenrieder Thomas M. Gress Martin Steinkamp \\ Division of Gastroenterology and Endocrinology, IBD Centre, Philipps University \\ Marburg, Marburg, Germany
}

\section{Key Words}

Ulcerative colitis · Disseminated pyoderma gangrenosum · Infliximab

\begin{abstract}
Pyoderma gangrenosum (PG) is an immune-mediated inflammatory skin condition representing one of the most distinct extraintestinal manifestations of inflammatory bowel disease (IBD). PG occurs independently from intestinal disease activity in about 1$2 \%$ of patients suffering from ulcerative colitis or Crohn's disease and is characterized by chronic deep skin ulcers whose exact pathogenesis is still unknown. So far, patients with ulcerative colitis have only been reported to develop PG during the course of IBD but not at the initial manifestation of bowel symptoms. This is the first report demonstrating the simultaneous onset of ulcerative colitis and severe multifocal PG. In addition, we provide first evidence that infliximab may have a particularly powerful effect in early disseminated PG compared to late-onset PG, advocating an early application of this drug.
\end{abstract}

\section{Introduction}

Pyoderma gangrenosum (PG) is an immune-mediated inflammatory skin condition occurring in about $1-2 \%$ of patients suffering from ulcerative colitis or Crohn's disease [1]. The skin alterations are characterized by painful deep ulcers rapidly developing from small pustules and often triggered by a minor injury, a sequence known as pathergy. PG normally shows well-defined erythematous to violaceous borders and usually appears on the lower limbs. Swabs of the wound initially prove to be sterile and histopathologic features are characterized by unspecific massive neutrophilic infiltration, hemorrhage and necrosis of the epidermis. 
PG is usually associated with autoimmune diseases such as rheumatoid arthritis and most commonly inflammatory bowel disease (IBD), although PG can be mimicked by other autoimmune and hematological disorders such as polyarteritis nodosa, Wegener's granulomatosis, antiphospholipid syndrome and lymphoma [2]. Furthermore, PG can also be triggered by several cytokines, growth factors and medications including granulocyte colony-stimulating factor [3], interferons [4] and antipsychotic drugs [5].

The prevailing therapeutic strategy is based on systemic immunosuppression using either corticosteroids, ciclosporin or infliximab, a monoclonal antibody against tumor necrosis factor $\alpha$ (TNF- $\alpha$ ). However, management of PG is mainly based on clinical experience and skin lesions often turn out to be refractory to standard treatment. Due to small case numbers and the lack of large randomised prospective trials, no guidelines for its treatment have been established to date.

Interestingly, patients with ulcerative colitis have only been reported to develop PG during the course of IBD but not during initial manifestation of bowel symptoms. We now report the first case of a simultaneous onset of ulcerative colitis and severe multifocal PG.

\section{Case Report}

Medical History

A 37-year-old male patient was transferred to our department from a small community hospital with an obscure septic clinical picture showing disseminated skin ulcerations associated with bloody, watery diarrhea for 3 weeks. Stool frequency was 6-10/day occurring during night and day and independently from food intake. Simultaneously to the gastrointestinal symptoms, several pustules with a surrounding inflammatory erythema developed on the back (fig. 1), chest, thigh and lower legs. These lesions (in total 10) rapidly progressed to large and painful cutaneous ulcerations, the largest of about $10 \times 9.5 \mathrm{~cm}$ on the left thigh (fig. 2a). Furthermore, the patient complained of arthralgia in the right knee. Further physical examination was without any pathological findings, there was no hint of any stool abnormalities in the past medical history, no fever, no night sweats, no previous dermatological diseases. The patient had lost about $6 \mathrm{~kg}$ during the last month. He denied taking any medication.

\section{Diagnostic Procedures}

Relevant Blood Analysis. Leukocytes: $11.4 \times 10^{9} / 1$ (4.3-10); erythrocytes: $4.0 \times 10^{12} / 1(4.5-6.0)$; hemoglobin: 102 g/l (140-180); MCV: 82 fl (84-97); MCH: 26 pg (27-32); MCHC: 315 g/l/Ery (320360); platelets: $624 \times 10^{9} / 1(150-350)$; hematocrit: $32 \%$ (47-53); C-reactive protein: $172 \mathrm{mg} / \mathrm{l}(<5)$; albumin: $15 \mathrm{~g} / \mathrm{l}(35-48)$.

Stool Tests. An infectious cause of the diarrhea was excluded by extensive stool tests including negative results for Clostridium difficile, E. coli, Shigella, Campylobacter, Salmonella, Yersinia and parasites.

Colonoscopy. Digital rectal examination: firm sphincter tonus, otherwise normal. Intubation of the terminal ileum showing normal mucosa. Hemorrhagic, edematous, friable and ulcerated mucosa with pseudopolyps involving the whole colon. Rectum in inversion normal with the exception of grade I hemorrhoids.

Histopathology. Microscopic examination of the colon mucosa demonstrated characteristic features of ulcerative colitis with distorted crypt architecture, inflammatory cell infiltrates, goblet cell depletion, mucosal ulcers and crypt abscesses. Cytomegalovirus was not detectable in biopsies from the colon, neither by microscopic examination nor through polymerase chain reaction.

\section{Therapy and Course of Disease}

An infectious cause of the diarrhea was excluded by extensive stool tests including negative results for Clostridium difficile, E. coli, Shigella, Campylobacter, Salmonella, Klebsiella, Yersinia and parasites. Colonoscopy and microscopic evaluation of multiple biopsies conclusively showed the characteristic picture of ulcerative colitis. The deep erythematous skin lesions initially proved to be sterile with central 
necrosis and well defined violaceous undermined edges. Consequently, the diagnosis of PG was made, and the biopsy was consistent with the diagnosis. For visualization of the whole gastrointestinal tract we additionally performed a gastroscopy as well as a MR enteroclysis showing no relevant pathological features.

Consequently, the patient was initially treated with high-dose prednisolone i.v. $(60 \mathrm{mg} /$ day $)$ and azathioprine $(2 \mathrm{mg} / \mathrm{kg} /$ day $)$. Two weeks later gastrointestinal symptoms as well as arthralgia ceased. However, ulcerations of the skin showed no tendency to heal despite topical tacrolimus ointment, daily dressing and wound cleansing. Therefore, infliximab infusion at a dosage of $5 \mathrm{mg} / \mathrm{kg}$ was administered and repeated at weeks 2 and 6 to treat the skin ulcers. Starting two weeks after the first infusion, lesions showed marked signs of improvement and disappeared four weeks later (fig. 2b). Infliximab infusions were well tolerated without any typical adverse effects. Prior to the first infliximab infusion active tuberculosis was ruled out by chest X-ray and skin test.

We were able to completely taper off prednisolone while azathioprine was continued $(2 \mathrm{mg} / \mathrm{kg} / \mathrm{day})$ to maintain remission of disease. The patient is closely monitored in our IBD outpatient clinic and remains in full remission. So far, he has not required additional infliximab infusions.

\section{Discussion}

PG is an immune-mediated inflammatory skin condition with unknown pathogenesis. The diagnosis of PG is primarily based on the typical clinical presentation showing deep and often painful skin ulcers with well-defined erythematous to violaceous borders and undermined edges, predominantly located on the lower extremities and the trunk. IBD is the most common underlying disorder and is found in $15-20 \%$ of PG cases [1]. Of those with a chronic course of ulcerative colitis, more than $6 \%$ may develop PG independent from the presence or absence of acute intestinal inflammation [6]. In these cases, intravenous application of infliximab, a chimeric monoclonal TNF- $\alpha$ antibody, has become an attractive and very effective treatment option [7]. A first randomised, doubleblind, placebo-controlled trial for the treatment of PG has recently underlined the therapeutic impact of infliximab [8]. That study included patients with PG irrespective of whether they had associated IBD. After administration of a single-shot infliximab infusion ( $5 \mathrm{mg} / \mathrm{kg}$ ), $46 \%$ of the infliximab group improved within two weeks as compared to $6 \%$ of patients in the placebo group. Interestingly, in another recent investigation by Regueiro et al. even higher remission rates were reported, most likely due to increased number of infliximab infusions administered [9]. In that case series, patients received a median of eight infusions of infliximab (range 1-24), starting with an induction dosing regimen at week 0 , week 2 and week 6 . Therefore, a sequential administration of infliximab appears superior, although the optimal number of infliximab infusion for induction of healing and sustained suppression of PG in patients with Crohn's disease or ulcerative colitis remains to be determined. Notably, patients included in that study had the diagnosis of IBD for at least 6 years, the earliest PG was diagnosed 5 years after the initial diagnosis and lasted 4 month before treatment with infliximab was started [9]. Interestingly, complete healing of PG, even of small skin lesions, took considerably longer and required additional infliximab infusions every $4-12$ weeks to maintain the clinical response in 10 of 13 patients. In contrast, we started infliximab therapy in our patient immediately after the initial diagnosis of PG. We observed complete healing of all skin ulcers after six weeks (two infliximab infusions) and until now, there has been no sign of PG recurrence or intestinal inflammation, suggesting a particularly powerful effect of infliximab at early stages of PG.

In summary, our case report is dealing with two interesting and novel aspects regarding the onset and therapy of PG in patients with ulcerative colitis. To the best of our knowledge, this is the first report demonstrating that disseminated PG can be present 
at the very first onset of ulcerative colitis. In fact, our patient had no history of diarrhea or extraintestinal symptoms prior to the onset of the reported episode of bloody diarrhea and multilocalized skin ulcers. In addition, we provide first evidence that infliximab may have a particularly powerful effect in early disseminated PG compared to late-onset PG, advocating an early application of this drug.

Fig. 1. Disseminated PG on the back.

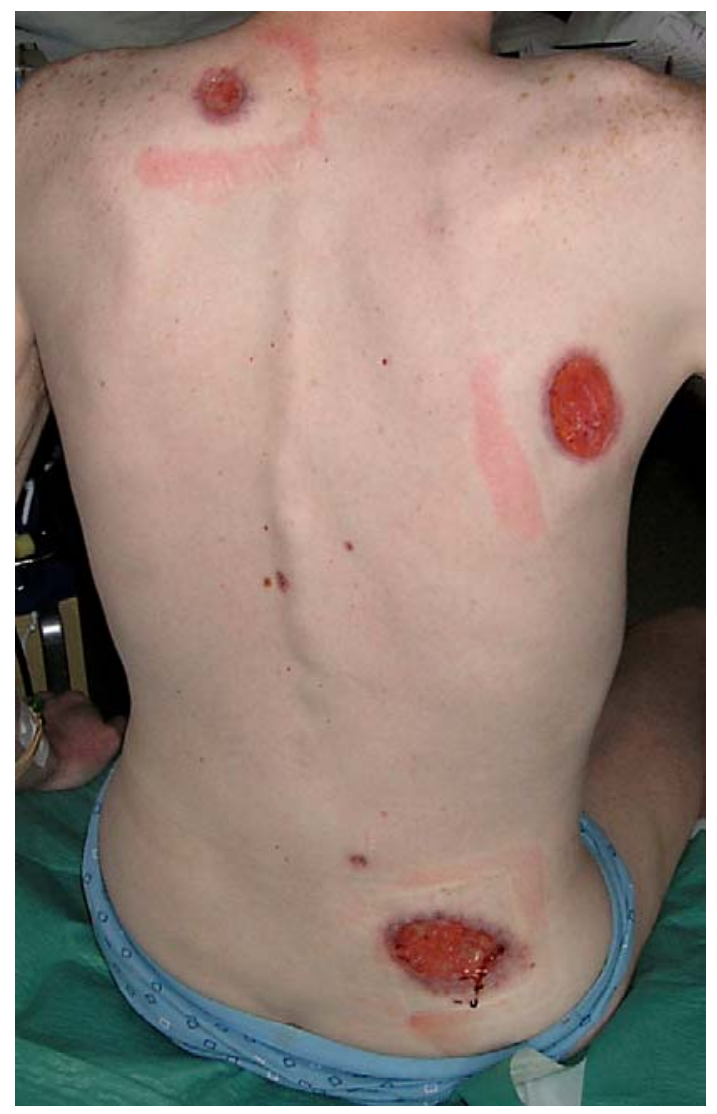


Fig. 2. a PG $(10 \times 9.5 \mathrm{~cm})$ on the left thigh with central necrosis and well-defined violaceous undermined edges. b Evolution of the same lesion 6 weeks after administration of two infliximab infusions of $5 \mathrm{mg} / \mathrm{kg}$ at week 0 and week 2: cribriform scarring.

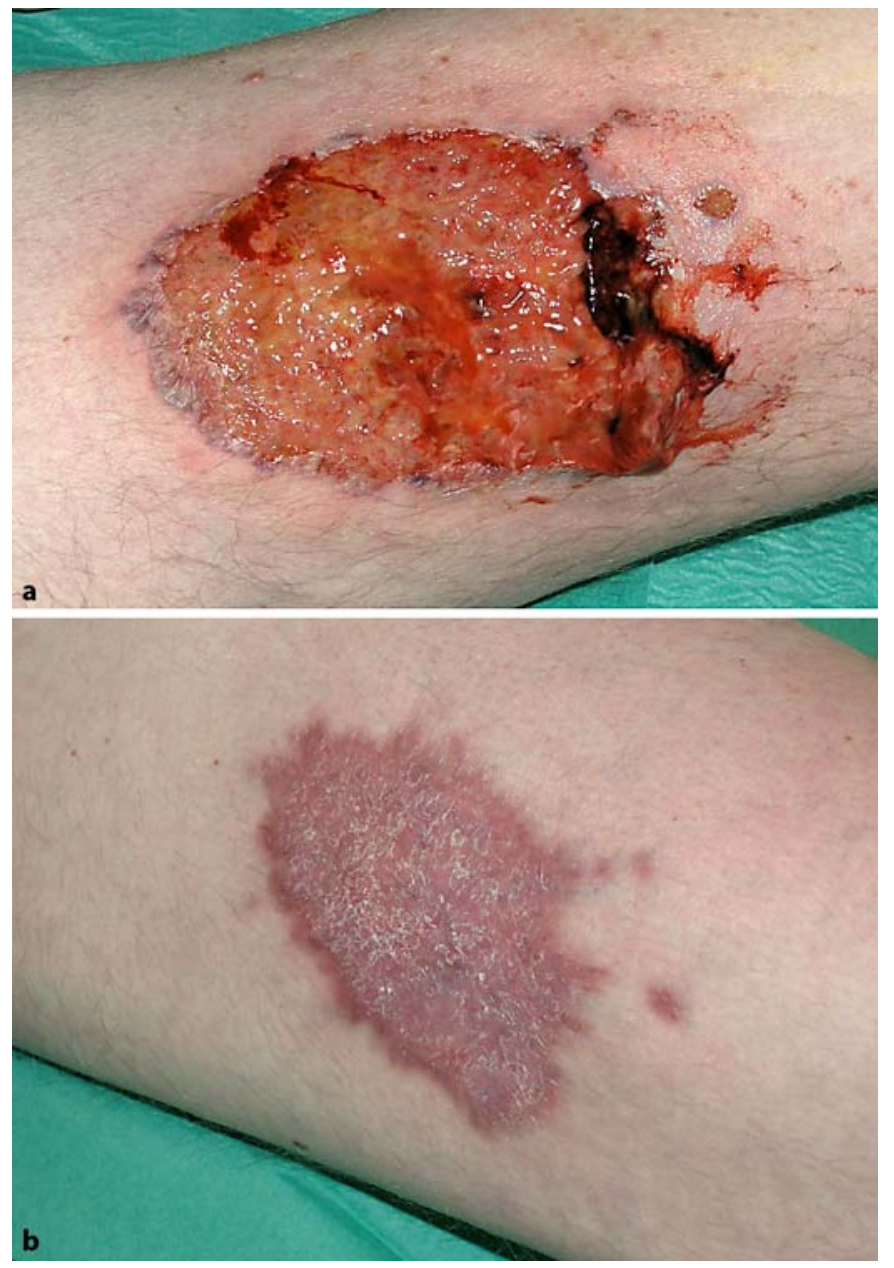




\section{References}

1 Callen JP: Pyoderma gangrenosum. Lancet 1998;351:581-585.

2 Nguyen KH, Miller JJ, Helm KF: Case reports and a review of the literature on ulcers mimicking pyoderma gangrenosum. Int J Dermatol 2003;42:84-94.

3 Ross HJ, Moy LA, Kaplan R, Figlin RA: Bullous pyoderma gangrenosum after granulocyte colony-stimulating factor treatment. Cancer 1991;68:441-443.

4 Montoto S, Bosch F, Estrach T, Blade J, Nomdedeu B, Nontserrat E: Pyoderma gangrenosum triggered by alpha2b-interferon in a patient with chronic granulocytic leukemia. Leuk Lymphoma 1998;30:199-202.

5 Srebrnik A, Shachar E, Brenner S: Suspected induction of a pyoderma gangrenosum-like eruption due to sulpiride treatment. Cutis 2001;67:253-256.

6 Langholz E: Ulcerative colitis: An epidemiological study based on a regional inception cohort, with special reference to disease course and prognosis. Dan Med Bull 1999;46:400-415.

7 Reichrath J, Bens G, Bonowitz A, Tilgen W: Treatment recommendations for pyoderma gangrenosum: An evidence-based review of the literature based on more than 350 patients. J Am Acad Dermatol 2005;53:273-283.

8 Brooklyn TN, Dunnill MG, Shetty A, Bowden JJ, Williams JD, Griffiths CE, Forbes A, Greenwood R, Probert CS: Infliximab for the treatment of pyoderma gangrenosum: A randomised, double blind, placebo controlled trial. Gut 2006;55:505-509.

9 Regueiro M, Valentine J, Plevy S, Fleisher MR, Lichtenstein GR: Infliximab for treatment of pyoderma gangrenosum associated with inflammatory bowel disease. Am J Gastroenterol 2003;98:1821-1826. 